ISSN-0253-7214

\title{
BIO-ECOLOGICAL STUDY OF FEW SPECIES OF APHIS LINN. IN NORTHEAST BIHAR AND THEIR ASSOCIATION WITH FOOD PLANTS AND NATURAL ENEMIES FOR POSSIBLE USE IN THE BIOLOGICAL CONTROL
}

\author{
Md. Equbal Ahmad, Sanjeev Kumar, Nishat Parween and Rakhshan \\ Aphid Systematics and Bio-control Laboratory, University Department of Zoology \\ T.M. Bhagalpur University, Bhagalpur- 812 007, Bihar, India \\ e-mail - equbal.tmbu@yahoo.com and nishatp744@gmail.com
}

\begin{abstract}
Aphids are small, soft bodied, polyphagic and polymorphic sap sucking insects. During the extensive survey of different localities of 10 districts of northeast Bihar in different seasons, 41 species of aphids were recorded on more than 122 plant species belong to 35 families. Among these, only 09 species of Aphis viz., Aphis affinis Del Guercio, Aphis craccivora Koch, Aphis fabae Scopoli, Aphis glycine Matsumura, Aphis gossypii Glover, Aphis nasturtii Kaltenbach, Aphis nerii Boyer de Fonscolombe, Aphis puniceae Passerini and Aphis spiraecola Patch were recorded in the target area. The maximum number of plants were infested by A. gossypii (46 plants), followed by A. craccivora (36 plants), A. nasturtii (18 plants) and A. spiraecola (18 plants).

Six species of coccinellids (Cheilomenes sexmaculata (Fabricius), Coccinella septempunctata Linnaeus, Coccinella transversalis Fabricius, Micraspis discolor (Fabricius), Pseudospidimerus circumflexa var. testaceus (Weise) and Scymnus pyrocheilus (Mulsant) and six species of syrphid predators (Allograpta javana (Wiedemann), Asarkina ericetorum (Fabricius), Episyrphus balteatus (DeGeer), Ischiodon scutellaris (Fabricius), Melanostoma orientale (Wiedemann) and Paragus serratus (Fabricius)) were recorded on Aphis spp. in the target area. Seven species of parasitoids viz, Aphelinus albipodus Fatima \& Hayat, Aphelinus basilicas Fatima \& Hayat, Aphelinus gossypii Timberlake, Aphelinus sp., Aphidius sp., Binodoxys indicus Subba Rao \& Sharma and Lipolexis oregmae (=scutellaris) (Gahan) were also recorded on Aphis spp. in the target area.

Six species of predators (C. sexmaculata, C. septempunctata, C. transversalis, E. balteatus, I. scutellaris and $P$. serratus) and three species of the parasitoids (A. gossypii, B. indicus and L. oregmae) were found abundantly on Aphis spp. in the target area and may be utilised in the biological control programme after further study.
\end{abstract}

KEYWORDS: Aphid, food plants, parasitoids, coccinellids, syrphids, predator

\section{INTRODUCTION}

Aphids are small, soft-bodied hemipteran bugs and most destructive pest of agriculture and horticulture crops. They increase their numbers very fast in favourable climatic condition due to parthenogenesis, viviparity and fast development 13. They attack all parts of plants and damage the crop directly by drawing sap from plant tissue9. They have also remarkable ability of transmitting plant viral diseases. Thus, the management of these aphids are essential to increase crop production $^{32}$. The biological control is one of the most important methods in Integrated Pest Management (IPM). ${ }^{32}$ The records of aphids along with their food plants, their parasitoids and predators in varying ecological conditions are essential to understand their relationships for the effective control measure. This source of information is considered as the first step 
in the biological control programme.

Several aphidiid parasitoids have been successfully used for the control of aphids. The active biological control attempts have been made by introduction of Diaeretiella rapae against the Diuraphis noxia ${ }^{\mathbf{1 8}}$. Lysiphlebus testides and Aphidius colemani were used successfully for the control of Pentalonia nigronervosa in South Pacific ${ }^{43}$. In India a lot of works have been done on different aspects of aphid parasitoids $\mathbf{3 , 7 , 8 , 1 7 , 3 6 , 3 7 , 3 9 , 4 1}$

Majority of coccinellid beetles are predaceous and play an important role in checking the population of many harmful insects such as aphids, coccids, scale insect, plant mites, thrips, leaf hoppers and other soft bodied insect. Several workers have surveyed the different parts of India and studied the coccinellid predators and their association with prey $\mathbf{1 , 1 2 , 2 1 , 2 4 , 2 5 , 2 6 , 2 7 , 2 8 , 3 5 , 3 8}$.

Syrphids are commonly known as hoverflies. The larvae of syrphid rank as the major natural enemies and play an important role in the suppression of aphid population ${ }^{15}$. Considerable amount of research has been conducted on the taxonomy, biology, ecology and feeding potential of syrphids by several workers $\mathbf{2 , 4 , 1 0}$, $16,19,20,22,23,29,30,31,34,42$

Northeast Bihar has rich agriculture lands where farmers face a lot of problems due to heavy infestation of crops by aphids. Thus, the extensive survey of the aphids along with their food plants in different seasons and their natural enemies are essential to understand their interrelationships for the effective control of economically important aphids. Keeping in view, the importance of bio-taxonomy and ecology, different localities of districts of Northeast Bihar, viz., Araria, Begusarai, Bhagalpur, Katihar, Kishanganj, Khagaria, Madhepura, Munger, Purnea and Saharsa were extensively surveyed in different seasons to obtain the records of the aphids, their parasitoids and predators to provide the raw materials (bio-agents) to evaluate their biotic potential foe the possible use in the biological control programme.

\section{MATERIALS AND METHOD}

Aphids, their parasitoids and predators (coccinellids and syrphids) were collected from different agricultural and horticultural plants from different localities of most of the districts of Northeast Bihar. Collected aphids were preserved in 70\% ethyl alcohol and glycerine (5:1) for taxonomical study. The systematical study of aphids was carried out by observing various taxonomical characters as given by ${ }^{1,11,33}$. Live aphids along with the food plants parts as well as the mummies were put into a translucent plastic vials $(2.5 \times 10 \mathrm{~cm})$ with wetted cotton at the bottom for the emergence of the parasitoids. Parasitoids were preserved in $70 \%$ alcohol for identification. Parasitoids were directly identified on the basis of Stary and Ghosh. ${ }^{40}$

Collected coccinellid larvae were kept in glass jar $(25 \times 10 \mathrm{~cm})$ with particular fresh aphids and reared in the laboratory till the 
emergence of adults. Taxonomical study of syrphids was done by observing various taxonomical characters mentioned by Dutta et. al. ${ }^{14}$. Few predators were sent to Zoological Survey of India, Kolkata for identification and confirmation.

\section{RESULTS AND DISCUSSION}

During the extensive survey of different localities of 10 districts of northeast Bihar in different seasons, 41 species of aphids on more than 122 plant species, 7 species of parasitoids, 6 species of coccinellids and 7 species of syrphids were recorded in the target area. Earlier, preliminary work has been done in few localities of this target area on aphids and their parasitoids ${ }^{3-8}$.

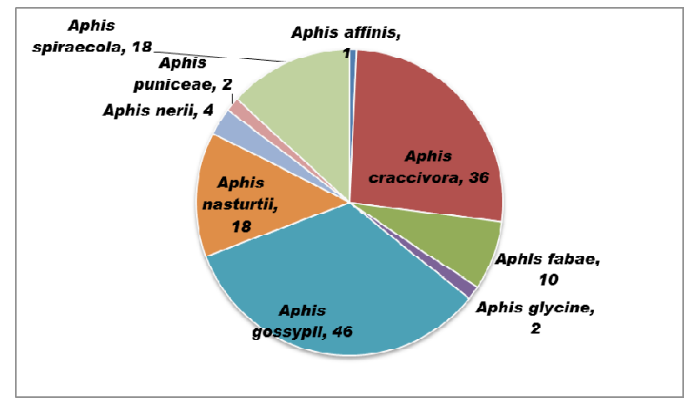

Fig. 1- Number of host plants infested by Aphis spp.

Among these, only 09 species of Aphis viz., Aphis affinis Del Guercio, Aphis craccivora Koch, Aphis fabae Scopoli, Aphis glycine Matsumura, Aphis gossypii Glover, Aphis nasturtii Kaltenbach, Aphis nerii Boyer de Fonscolombe, Aphis puniceae Passerini and Aphis spiraecola Patch were recorded in the target area (Fig.-1). The maximum number of plants were infested by A. gossypii (46 plants), followed by $A$. craccivora (36 plants), A. nasturtii (18 plants) and A. spiraecola (18 plants).

Six species of coccinellids (Cheilomenes sexmaculata (Fabricius), Coccinella septempunctata Linnaeus, Coccinella transversalis Fabricius, Micraspis discolor (Fabricius), Pseudospidimerus circumflexa var. testaceus (Weise) and Scymnus pyrocheilus (Mulsant) and six species of syrphid predators (Allograpta javana (Wiedemann), Asarkina ericetorum (Fabricius), Episyrphus balteatus (DeGeer), Ischiodon scutellaris (Fabricius), Melanostoma orientale (Wiedemann) and Paragus serratus (Fabricius)) were recorded on Aphis spp. in the target area.

These aphids were found to be parasitized by seven species of parasitoids viz., Aphelinus albipodus Fatima \& Hayat, Aphelinus basilicas Fatima \& Hayat, Aphelinus gossypii Timberlake, Aphelinus sp., Aphidius sp., Binodoxys indicus Subba Rao \& Sharma and Lipolexis oregmae (=scutellaris) (Gahan) in the target area (Table-1).

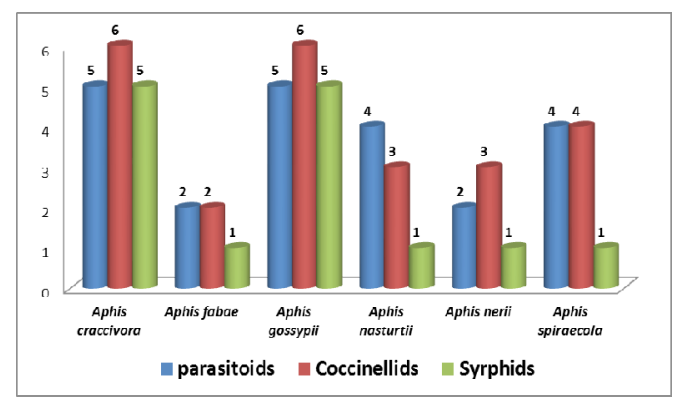

Fig.-2: Number of parasitoids and predators (coccinellids \& syrphids) recorded on important Aphis spp. 
Table -1 Association of few species of Aphis with their food plants and natural enemies

\begin{tabular}{|c|c|c|c|}
\hline Aphids /Plants/ Family & $\begin{array}{l}\text { Coccinellids/Intensity } \\
\text { of predation }\end{array}$ & $\begin{array}{l}\text { Syrphids/Intensity } \\
\text { of predation }\end{array}$ & $\begin{array}{c}\text { Parasitoids/Intensity } \\
\text { of parasitisation }\end{array}$ \\
\hline \multicolumn{4}{|l|}{ 1. APHIS AFFINIS } \\
\hline 1. Mentha spicata (Labiateae) & - & - & - \\
\hline \multicolumn{4}{|l|}{ 2. APHIS CRACCIVORA } \\
\hline $\begin{array}{l}\text { 1. Abelmoschus esculentus } \\
\text { (Malvaceae) }\end{array}$ & - & - & $\begin{array}{ll}\text { A.gossypii } & + \\
\text { B. indicus } & ++\end{array}$ \\
\hline $\begin{array}{l}\text { 2. Ageratum conyzoides } \\
\text { (Asteraceae) }\end{array}$ & - & - & $\begin{array}{ll}\text { A. gossypii } & ++ \\
\text { B.indicus } & ++++ \\
\text { L. oregmae } & +++\end{array}$ \\
\hline $\begin{array}{l}\text { 3. Brassica. oleracea var. } \\
\text { botrytis (Brassicaceae) }\end{array}$ & - & - & A.gossypii \\
\hline 4. Cajanus cajan (Fabaceae) & $\begin{array}{l}\text { C. } \text { sexmaculata }++ \\
\text { S. pyrocheilus }++\end{array}$ & E. balteatus ++ & $\begin{array}{ll}\text { A. gossypii } & +++ \\
\text { B. indicus } & ++++ \\
\text { L. oregmae } & +++ \text {. }\end{array}$ \\
\hline $\begin{array}{l}\text { 5. Hibiscus sabdariffa } \\
\text { (Malvaceae) }\end{array}$ & & & L. oregmae + \\
\hline $\begin{array}{l}\text { 6. Lablab purpureus } \\
\text { (Fabaceae) }\end{array}$ & $\begin{array}{l}\text { C. } \text { sexmaculata }+++ \\
\text { C. septempuctata }++ \\
\text { M. discolor }+++ \\
\text { P. circumflexa }++++ \\
\text { S. pyrocheilus }++\end{array}$ & $\begin{array}{l}\text { A. javana }+ \\
\text { A. ericetorum }++ \\
\text { E. balteatus }++ \\
\text { I. scutellaris }+++ \\
\text { P. serratus }+++\end{array}$ & $\begin{array}{ll}\text { A.albipodus } & ++++ \\
\text { A. basilicus } & ++ \\
\text { A. gossypii } & ++++ \\
\text { B. indicus } & ++++ \\
\text { L. oregmae } & ++++\end{array}$ \\
\hline $\begin{array}{l}\text { 7. Lagenaria siceraria } \\
\text { (= Lageneria vulgaris) } \\
\text { (Cucurbitaceae) }\end{array}$ & - & - & $\begin{array}{ll}\text { A. } \text { gossypii } & ++++ \\
\text { B. indicus } & ++++ \\
\text { L. oregmae } & ++++\end{array}$ \\
\hline 8. Melilotus alba (Fabaceae) & - & - & B. indicus \\
\hline $\begin{array}{l}\text { 9. Moringa oleifera } \\
\text { (Morigiaceae) }\end{array}$ & & & L. oregmae + \\
\hline $\begin{array}{l}\text { 10. Phaseolus sinensis } \\
\text { (Fabaceae) }\end{array}$ & $\begin{array}{l}\text { C. } \text { sexmaculata }++ \\
\text { C. septempuctata }+++ \\
\text { S. pyrocheilus }++\end{array}$ & $\begin{array}{l}\text { I. } \text { scutellaris }+++ \\
\text { P. } \text { serratus }+++\end{array}$ & $\begin{array}{ll}\text { B. } \text { indicus } & ++ \\
\text { L. } \text { oregmae } & ++++\end{array}$ \\
\hline $\begin{array}{l}\text { 11. Trigonella foenumgraecum } \\
\text { (Fabaceae) }\end{array}$ & - & - & B. indicus \\
\hline 12. Vicia faba (Fabaceae) & C. sexmaculata + & - & $\begin{array}{ll}\text { A. } \text { gossypii } & +++ \\
\text { B. } \text { indicus } & ++ \\
\text { L. oregmae } & ++++\end{array}$ \\
\hline $\begin{array}{l}\text { 13. Vigna mungo var. mungo } \\
\text { (Fabaceae) }\end{array}$ & $\begin{array}{l}\text { C. } \text { transversalis }++ \\
\text { S. pyrocheilus ++ }\end{array}$ & $\begin{array}{l}\text { I. } \text { scutellaris }++ \\
P . \text { serratus }++\end{array}$ & $\begin{array}{ll}\text { B. } \text { indicus } & ++ \\
\text { L. } \text { oregmae } & +++\end{array}$ \\
\hline
\end{tabular}




\begin{tabular}{|c|c|c|c|c|}
\hline 14. Vigna sativa (Fabaceae) & - & - & B. indicus & + \\
\hline \multicolumn{5}{|l|}{ 3. APHIS FABAE } \\
\hline $\begin{array}{l}\text { 1. Lagenaria siceraria } \\
\text { (Cucurbitaceae) }\end{array}$ & $\begin{array}{l}\text { C. } \text { sexmaculata }++ \\
\text { S. pyrocheilus }++\end{array}$ & - & $\begin{array}{l}\text { B. } \text { indicus } \\
\text { L. oregmae }\end{array}$ & $\begin{array}{l}++ \\
++\end{array}$ \\
\hline $\begin{array}{l}\text { 2. Luffa cylindrica } \\
\text { (Cucurbitaceae) }\end{array}$ & C. septempuctata ++ & I. scutellaris +++ & B. indicus & ++ \\
\hline \multicolumn{5}{|l|}{ 4. APHIS GLYCINE } \\
\hline $\begin{array}{l}\text { 1. Catheranthus roseus } \\
\text { (Apocynaceae) }\end{array}$ & - & - & - & \\
\hline $\begin{array}{l}\text { 2. Lycopersicon esculentum } \\
\text { (Solanaceae) }\end{array}$ & - & - & - & \\
\hline \multicolumn{5}{|l|}{ 5. APHIS GOSSYPII } \\
\hline $\begin{array}{l}\text { 1. Abelmoschus esculentus } \\
\text { (Malvaceae) }\end{array}$ & $\begin{array}{l}\text { C. } \text { sexmaculata }+++ \\
\text { C. } \text { septempuctata }+++ \\
\text { C. transversalis }++ \\
\text { S. } \text { pyrocheilus }++\end{array}$ & - & $\begin{array}{l}\text { A. gossypii } \\
\text { L. oregmae }\end{array}$ & $\begin{array}{l}+ \\
++++\end{array}$ \\
\hline $\begin{array}{l}\text { 2. Ageratum conyzoides } \\
\text { (Asteraceae) }\end{array}$ & $\begin{array}{l}\text { C. } \text { transversalis }++ \\
\text { S. pyrocheilus }++\end{array}$ & P. serratus +++ & B. indicus & +++ \\
\hline 3. Cajanus cajan (Fabaceae) & $\begin{array}{l}\text { C. } \text { sexmaculata }+++ \\
\text { C. septempuctata }++\end{array}$ & $\begin{array}{l}\text { E. balteatus }++ \\
P . \text { serratus }+++\end{array}$ & $\begin{array}{l}\text { A. gossypii } \\
\text { B. indicus }\end{array}$ & $\begin{array}{l}++ \\
++++\end{array}$ \\
\hline $\begin{array}{l}\text { 4. Capsicum frutescens } \\
\text { (Solanaceae) }\end{array}$ & $\begin{array}{l}\text { C. } \text { sexmaculata }++ \\
\text { C. septempuctata }++ \\
\text { S. pyrocheilus }+++\end{array}$ & - & $\begin{array}{l}\text { A. gossypii } \\
\text { B. indicus } \\
\text { L. oregmae }\end{array}$ & $\begin{array}{l}+++ \\
+++ \\
+++\end{array}$ \\
\hline $\begin{array}{l}\text { 5. Catheranthus rosesus } \\
\text { (Aponogetonaceae) }\end{array}$ & $\begin{array}{l}\text { C. } \text { sexmaculata }++ \\
\text { S. pyrocheilus }++\end{array}$ & - & A. gossypii + & + \\
\hline 6. Citrus limon (Rutaceae) & - & - & B. indicus & ++ \\
\hline $\begin{array}{l}\text { 7. Clerodendrum infortunatum } \\
\text { (Verbanaceae) }\end{array}$ & - & - & $\begin{array}{l}\text { B. indicus } \\
\text { L. oregmae }\end{array}$ & $\begin{array}{l}+++ \\
++\end{array}$ \\
\hline $\begin{array}{l}\text { 8. Coccinia indica } \\
\text { (Cucurbitaceae) }\end{array}$ & C. transversalis ++ & I. scutellaris +++ & $\begin{array}{l}\text { B. indicus } \\
\text { L. oregmae }\end{array}$ & $\begin{array}{l}++ \\
++++\end{array}$ \\
\hline 9. Colocasia sp. (Araceae) & S. pyrocheilus + & - & $\begin{array}{l}\text { Aphelius sp. } \\
\text { L. oregmae }\end{array}$ & $\begin{array}{l}++ \\
+++\end{array}$ \\
\hline 10. Croton sp. (Euphorbiaceae) & S. pyrocheilus ++ & I. scutellaris + & & \\
\hline $\begin{array}{l}\text { 11. Cucurbita hispida } \\
\text { (Cucurbitaceae) }\end{array}$ & - & - & B. indicus & +++ \\
\hline 12. Foeniculum vulgare & C. sexmaculata ++ & - & - & \\
\hline 13.Helicteris isora & S. pyrocheilus ++ & - & - & \\
\hline
\end{tabular}




\begin{tabular}{|c|c|c|c|c|}
\hline $\begin{array}{l}\text { 14. Hibiscus rosasinensis } \\
\text { (Malvaceae) }\end{array}$ & $\begin{array}{l}\text { C. sexmaculata }++++ \\
\text { S. pyrocheilus }+++\end{array}$ & $\begin{array}{l}\text { I. } \text { scutellaris }+++^{++} \\
\text {P.serratus }++\end{array}$ & $\begin{array}{l}\text { B. indicus } \\
\text { L. oregmae }\end{array}$ & \\
\hline $\begin{array}{l}\text { 15. Hibiscus sabdariffa } \\
\text { (Malvaceae) }\end{array}$ & - & - & B. indicus & ++ \\
\hline 16. Hordeum vulgare (Poaceae) & - & - & B. indicus & + \\
\hline $\begin{array}{l}\text { 17. Impatiens balsamina } \\
\text { (Balsaminaceae) }\end{array}$ & - & - & \multicolumn{2}{|c|}{ Aphelinus sp.++ } \\
\hline $\begin{array}{l}\text { 18. Lablab purpureus } \\
\text { (Fabaceae) }\end{array}$ & - & - & $\begin{array}{l}\text { A. gossypii } \\
\text { B. indicus }\end{array}$ & $\begin{array}{l}++ \\
+++\end{array}$ \\
\hline $\begin{array}{l}\text { 19. Lagenaria siceraria } \\
\text { (Cucurbitaceae) }\end{array}$ & $\begin{array}{l}\text { C. sexmaculata }++++ \\
\text { S. pyrocheilus }++\end{array}$ & $\begin{array}{l}\text { A. javana }+++ \\
\text { A. ericetorum }+++ \\
\text { I. scutellaris }++++ \\
\text { P.serratus }+++\end{array}$ & $\begin{array}{l}\text { A. gossypii } \\
\text { B. indicus } \\
\text { L. oregmae }\end{array}$ & $\begin{array}{l}++ \\
++++ \\
++\end{array}$ \\
\hline 20. Lawsonia inermis & S. pyrocheilus + & - & $\begin{array}{l}\text { B. indicus } \\
\text { L. oregmae }\end{array}$ & $\begin{array}{l}++ \\
++\end{array}$ \\
\hline $\begin{array}{l}\text { 21. Luffa acutangula } \\
\text { (Cucurbitaceae) }\end{array}$ & & & A. gossypii & +++ \\
\hline $\begin{array}{l}\text { 22. Luffa cylindrical } \\
\text { (Cucurbitaceae) }\end{array}$ & C. sexmaculata ++++ & I. scutellaris ++++ & $\begin{array}{l}\text { A. gossypii } \\
\text { B. indicus } \\
\text { L. oregmae }\end{array}$ & $\begin{array}{l}+++ \\
++++ \\
++\end{array}$ \\
\hline $\begin{array}{l}\text { 23. Lycopersicon esculantum } \\
\text { (Solanaceae) }\end{array}$ & $\begin{array}{l}\text { C. septempuctata ++ } \\
\text { S. pyrocheilus ++ }\end{array}$ & - & B. indicus & ++ \\
\hline $\begin{array}{l}\text { 24. Momordica charantia } \\
\text { (Cucurbitaceae) }\end{array}$ & $\begin{array}{l}\text { C. } \text { sexmaculata }+++ \\
\text { C. } \text { septempuctata }+ \\
\text { C. transversalis }++ \\
\text { S. } \text { pyrocheilus }++\end{array}$ & I. scutellaris ++ & $\begin{array}{l}\text { A. gossypii } \\
\text { B. indicus } \\
\text { L. oregmae }\end{array}$ & $\begin{array}{l}++++ \\
++ \\
+++\end{array}$ \\
\hline 25. Ocimum sanctum & S. pyrocheilus ++ & - & $\begin{array}{l}\text { A. gossypii } \\
\text { B. indicus } \\
\text { L. oregmae }\end{array}$ & $\begin{array}{l}+ \\
++++ \\
+\end{array}$ \\
\hline $\begin{array}{l}\text { 26. Psidium guajava } \\
\text { (Myrtaceae) }\end{array}$ & $\begin{array}{l}\text { C. } \text { septempuctata }+ \\
\text { P. circum flexa }++ \\
\text { S. pyrocheilus }+++\end{array}$ & - & $\begin{array}{l}\text { B. indicus } \\
\text { L. oregmae }\end{array}$ & $\begin{array}{l}++ \\
+\end{array}$ \\
\hline 27. Rosa sp. (Rosaceae) & S. pyrocheilust & - & $\begin{array}{l}\text { B. indicus } \\
\text { L. oregmae }\end{array}$ & $\begin{array}{l}+++ \\
+\end{array}$ \\
\hline $\begin{array}{l}\text { 28. Solanum melongena } \\
\text { (Solanaceae) }\end{array}$ & $\begin{array}{l}\text { C. } \text { sexmaculata }+++ \\
\text { S. pyrocheilus }++\end{array}$ & - & $\begin{array}{l}\text { A. albipodus } \\
\text { B. indicus } \\
\text { L. oregmae }\end{array}$ & $\begin{array}{l}+ \\
++++ \\
++++\end{array}$ \\
\hline $\begin{array}{l}\text { 29. Solanum tuberosum } \\
\text { (Solanaceae) }\end{array}$ & - & - & B. indicus & +++ \\
\hline
\end{tabular}




\begin{tabular}{|c|c|c|c|c|}
\hline 30. Tagetes sp. (Asteraceae) & S. pyrocheilus ++ & - & $\begin{array}{l}\text { A. gossypii } \\
\text { B. indicus }\end{array}$ & $\begin{array}{l}+ \\
++\end{array}$ \\
\hline \multicolumn{5}{|l|}{ 6. APHIS NASTURTII } \\
\hline $\begin{array}{l}\text { 1. Ageratum conyzoides } \\
\text { (Asteraceae) }\end{array}$ & $\begin{array}{l}\text { C. transversalis }++ \\
\text { S. pyrocheilus }++\end{array}$ & & & \\
\hline $\begin{array}{l}\text { 2. Capsicum frutescens } \\
\text { (Solanaceae) }\end{array}$ & $\begin{array}{l}\text { P. } \text { circumflexa }++++ \\
\text { S. pyrocheilus }+++\end{array}$ & - & $\begin{array}{l}\text { B. indicus } \\
\text { L. oregmae }\end{array}$ & $\begin{array}{l}++++ \\
+++\end{array}$ \\
\hline $\begin{array}{l}\text { 3. Chrysanthemum indicum } \\
\text { (Asteraceae) }\end{array}$ & - & - & $\begin{array}{l}\text { Aphidius sp. } \\
\text { L. oregmae }\end{array}$ & $\begin{array}{l}++ \\
+++\end{array}$ \\
\hline $\begin{array}{l}\text { 4. Cucurbita hispida } \\
\text { (Cucurbitaceae) }\end{array}$ & - & - & $\begin{array}{l}\text { B. indicus } \\
\text { L. oregmae }\end{array}$ & $\begin{array}{l}++ \\
+++\end{array}$ \\
\hline 5. Ficus religiosa (Moraceae) & - & - & $\begin{array}{l}\text { B. indicus } \\
\text { L. oregmae }\end{array}$ & $\begin{array}{l}+++ \\
++\end{array}$ \\
\hline $\begin{array}{l}\text { 6. Hibuscus sabdariffa } \\
\text { (Malvaceae) }\end{array}$ & - & - & L. oregmae & ++ \\
\hline $\begin{array}{l}\text { 7. Lagenaria siceraria } \\
\text { (Cucurbitaceae) }\end{array}$ & S. pyrocheilus ++ & A. ericetorum +++ & $\begin{array}{l}\text { B. indicus } \\
\text { L. oregmae }\end{array}$ & $\begin{array}{l}+++ \\
+++\end{array}$ \\
\hline $\begin{array}{l}\text { 8. Luffa cylindrical } \\
\text { (Cucurbitaceae) }\end{array}$ & - & - & $\begin{array}{l}\text { B. indicus } \\
\text { L. oregmae }\end{array}$ & $\begin{array}{l}++ \\
+++\end{array}$ \\
\hline $\begin{array}{l}\text { 9. Lycopersicon esculentum } \\
\text { (Solanaceae) }\end{array}$ & - & - & $\begin{array}{l}\text { B. indicus } \\
\text { L. oregmae }\end{array}$ & $\begin{array}{l}++ \\
++++\end{array}$ \\
\hline $\begin{array}{l}\text { 10. Psidium guajava } \\
\text { (Myrtaceae) }\end{array}$ & - & - & B. indicus & ++++ \\
\hline $\begin{array}{l}\text { 11. Solanum melongena } \\
\text { (Solanaceae) }\end{array}$ & $\begin{array}{l}\text { C. } \text { sexmaculata }++ \\
\text { S. pyrocheilus }+++\end{array}$ & - & $\begin{array}{l}\text { B. indicus } \\
\text { L. oregmae }\end{array}$ & $\begin{array}{l}++++ \\
++++\end{array}$ \\
\hline $\begin{array}{l}\text { 12. Solanum tuberosum } \\
\text { (Solanaceae) }\end{array}$ & - & - & B. indicus & ++ \\
\hline 13. Tectona grandis & - & - & L. oregmae & +++ \\
\hline 7. APHIS NERII & & - & & \\
\hline 1. Calotropis gigantea & $\begin{array}{l}\text { C. } \text { sexmaculata }+++ \\
\text { C. } \text { septempuctata }++ \\
\text { S. pyrocheilus }++\end{array}$ & - & $\begin{array}{l}\text { B. indicus } \\
\text { L. oregmae }\end{array}$ & $\begin{array}{l}++++ \\
++\end{array}$ \\
\hline $\begin{array}{l}\text { 2. Coccinia indica } \\
\text { (Cucurbitaceae) }\end{array}$ & - & - & $\begin{array}{l}\text { B. indicus } \\
\text { L. oregmae }\end{array}$ & $\begin{array}{l}+++ \\
+++\end{array}$ \\
\hline 3. Nerium indicum & C. septempuctata ++ & P. serratus +++ & $\begin{array}{l}\text { B. indicus } \\
\text { L. oregmae }\end{array}$ & $\begin{array}{l}++ \\
++++\end{array}$ \\
\hline \multicolumn{5}{|l|}{ 8. APHIS PUNICAE } \\
\hline 1. Colocasia sp. (Araceae) & - & - & & \\
\hline
\end{tabular}

109 • J. Adv. Zool. 2020:41(1\&2) 


\begin{tabular}{|c|c|c|c|}
\hline $\begin{array}{l}\text { 2. Punica granatum } \\
\text { (Punicae) }\end{array}$ & - & - & \\
\hline \multicolumn{4}{|l|}{ 9. APHIS SPIRAECOLA } \\
\hline $\begin{array}{l}\text { 1. Ageratum conyzoides } \\
\text { (Asteraceae) }\end{array}$ & $\begin{array}{l}\text { C. } \text { transversalis ++ } \\
\text { S. pyrocheilus ++ }\end{array}$ & P. serratus ++ & $\begin{array}{l}\text { A. gossypii + } \\
\text { B. indicus }++++ \\
\text { L. oregmae }++++\end{array}$ \\
\hline 2. Cajanus cajan (Fabaceae) & - & - & B. indicus ++ \\
\hline $\begin{array}{l}\text { 3. Cestrum nocturnum } \\
\text { (Solanaceae) }\end{array}$ & - & - & B. indicus ++ \\
\hline 4. Chrysanthemum indicum & S. pyrocheilus ++ & - & $\begin{array}{l}\text { A. gossypii }+ \\
\text { Aphidius sp. }+\end{array}$ \\
\hline 5. Coriandrum sativum & C. sexmaculata +++ & - & $\begin{array}{l}\text { A. gossypii }+++ \\
\text { B. indicus }+++ \\
\text { L. oregmae }+\end{array}$ \\
\hline 6. Cosmos sp. & C. sexmaculata ++ & - & \\
\hline 7. Dahalia sp. & - & - & A. gossypii ++ \\
\hline $\begin{array}{l}\text { 8. Hibiscus sabdariffa } \\
\text { (Malvaceae) }\end{array}$ & - & - & B. indicus +++ \\
\hline $\begin{array}{l}\text { 9. Lagenaria siceraria } \\
\text { (Cucurbitaceae) }\end{array}$ & $\begin{array}{l}\text { C. } \text { septempuctata }++ \\
\text { S. pyrocheilus }++\end{array}$ & P. serratus +++ & - \\
\hline $\begin{array}{l}\text { 10. Solanum melongena } \\
\text { (Solanaceae) }\end{array}$ & C. sexmaculata ++ & - & - \\
\hline 11. Sonchus sp. & - & - & B. indicus + \\
\hline 12. Tagetes sp. (Asteraceae) & - & & $\begin{array}{ll}\text { A. gossypii }++++ \\
\text { B. indicus }+++ \\
\text { L. oregmae }+++\end{array}$ \\
\hline
\end{tabular}

Intensity of parasitisation/predation

$$
+=\text { low }++=\text { Moderate }+++=\text { High }++++=\text { Very High }
$$

Six species of predators $(C$. sexmaculata, $C$. septempunctata, $C$. transversalis, E. balteatus, I. scutellaris and $P$. serratus) and three species of the parasitoids (A. gossypii, B. indicus and $L$. oregmae) were found abundantly on Aphis spp.(Fig.-2) in the target area and may be utilised in the biological control programme after further study.

\section{APHIS AFFINIS DEL GUERCIO}

It is small dark grey-green aphid to almost black in colour. It was recorded in only one place in the locality.

Host plants : It forms colony on tender upper leaves of Mentha spicata of family Labiateae in the target area and moderate intensity of infestation was 
observed.

Seasonal abundance: It was recorded only in one locality during April.

Parasitoid : Not recorded.

Predator : Not recorded.

\section{APHIS CRACCIVORA KOCH}

Adult $A$. craccivora are shiny black in colour and immatures are light brown and lightly dusted with wax. It is commonly called cowpea aphid. It was recorded in all localities of the target area.

Host plants : A. craccivora is a most serious polyphagous pest in the target area. During the extensive survey, it was recorded on 36 species of host plants. Highly infested food plants were Abelmoschus esculentus, Cajanus cajan, Lablab purpureus, Phaseolus sinensis, Solanum xanthocarpum, Vicia faba, Vigna mungo var. mungo and Vigna sp. High to moderate infestation was also observed on several food plants viz., Cicer arietenum, Clerodendrum splendens, Hibiscus sabdariffa, Lagenaria siceraria, Lycopersicon esculentum, Mangifera indica, Melilotus alba, Mirabilis jalapa, Nerium oleander, Vigna radiata var. radiata, Solanum tuberosum and Trigenella foenumgraecum. However on rest of food plants, low infestation was observed (Brassica campestris, Brassica. oleracea var. botrytis, Bougainvillea sp., Citrus limon, Cucumis melo, Cyamopsis sp. Lathyrus aphaca, Lathyrus sativa, Pisum sativum ). The most suffered food plants belong to families Fabaceae.

Seasonal abundance : It was recorded in the month of August to May. Its peak population was observed during September to March. During May to July its population declined due to increase of temperature.

Parasitoids : A. albipodus, A. basilicas, A. gossypii, B. indicus and $L$. oregmae were recorded in the target area on this aphid with low to high rate of parasitisation (Table-1

\section{Predators :}

a. Coccinellids: All six species of coccinellid predators viz., C. sexmaculata, $C$. septempunctata, $C$. transversalis, $M$. discolor, P. circumflexa, S. pyrocheilus were recorded on this aphid with moderate to high rate of predation ( Table -1 ).

b. Syrphids : A. javana, A. ericetorum, E. balteatus, I. scutellaris and $P$. serratus were recorded in the target area on A. craccivora ( Table -1).

\section{APHIS FABAE}

A. fabae is commonly known as bean aphid. Young colonies consist of brown aphids were collected on young shoots in only few localities in the target area.

Host plants : In the present survey, it was recorded on 10 food plants viz., Brachycorne sp., Brassica oleracea var. botrytis, Chrysanthemum indicum, Cyamopsis tetragonolobus, Hibiscus rosasinensis, Lagenaria siceraria, Luffa cylindrica, Lycopersicon esculentum, Solanum tuberosum, Tagetes sp. Highly infested plants were Brachycorne sp., Chrysanthemum indicum, L. sciceraria, and Tegetes sp. The most suffered family was 


\section{Asteraceae.}

Seasonal abundance : A. fabae was started to build up its population during December. Its peak population was observed in the month of February (Table-1).

Parasitoids : $B$. indicus and $L$. oregmae were recorded in the target with moderate rate of parasitisation.

Predators :

a. Coccinellids: Only $C$. septempunctata and Scymnus pyrocheilus was recorded on this aphid with moderate rate of predation.

b. Syrphids : only I. scutellaris was recorded in the target area on A. fabae.

\section{APHIS GLYCINE}

It is commonly called soybean aphid. It was recorded only from two localities of the target area.

Host Plants : This aphid is not common in the target area and were recorded on only two plants Catheranthus roseus and Lycopersicon esculentum with low intensity of infestation.

Seasonal abundance : It was recorded during September to December.

Parasitoid : Not recorded.

Predator : Not recorded.

\section{APHIS GOSSYPII}

It is commonly known as cotton aphid or melon aphid. Individuals of the same colony show a great variation in size. $A$. gossypii was the most common aphid and recorded in all localities of the target area.
Host plants : A. gossypii is a most serious pest in the target area and was recorded on more than 46 food plants. The most suffered food plants were $A$. esculantus, Clerodendrum infortunatum, Capsicum frutescens, Coccinia indica, $L$. siceraria, Lawsonia inermis,, Momordica charantia, Ocimum tenuiflorum, Psidium guajava, Solanum melongena, S. tuberosum and Tagetes sp.

Seasonal abundance: A. gossypii is found throughout the year except in June due to high temperature. It started to appear during July. Its high intensity of infestation was observed during October to February on several economically important plants (Table-1).

Parasitoids : Five species of the parasitoids viz., A. albipodus A. gossypii, Aphilinus sp., B. indicus and $L$. oregmae were recorded. B. indicus and $L$. oregmae were found abundantly in most of the localities with moderate to high rate of parasitsation in the target area.

\section{Predators :}

a. Coccinellids: All six species of coccinellid viz., $C$. sexmaculata, $C$. septempunctata, $C$. transversalis, $M$. discolor, P. circumflexa, S. pyrocheilus were recorded on this aphid with moderate to high rate of predation ( Table -1 ).

b. Syrphids : A. javana, A. ericetorum, E. balteatus, I. scutellaris and $P$. serratus were recorded on this aphid (Table -1). 


\section{APHIS NASTURTII}

Distribution : A. nasturtii is also a common aphid observed in almost all localities of the target area.

Host plants : A. nasturtii is a polyphagous in nature and recorded on 18 food plants. The intensity of infestation was very low to very high on different plants in different localities. The highly infested plants were $A$. esculantus, $C$. frutescens, $C$. infortunatum, Ficus religiosa, $P$. guajava and $S$. melongena. Seasonal abundance: It is found mostly in months of last July to May in different localities of target area (Table-1). Its high infestation was observed in month of October to March on different food plants.

Parasitoids : Apidius sp., A. gossypii, $B$. indicus and $L$. oregmae were recorded in the target area with moderate to high rate of parasitisation on this aphid.

\section{Predators :}

a. Coccinellids: $C$. sexmaculata, $P$. circumflexa and $S$. pyrocheilus were recorded on this aphid with moderate to high rate of predation (Table -1).

b. Syrphids : only one species, $A$. ericetorum was recorded on this aphid (Table -1).

\section{APHIS NERII}

It is commonly known as nerium aphid. The aphid is very easy to identify because of its unique bright yellow-orange colour and dark brown appendages.

Distribution : It was recorded from several localities in the target area.

Host plants : A. nerii was recorded only on 4 food plants of different families (Table-1). The high intensity of infestation was recorded on Calotropis gigantea and Coccinia indica in different localities of target area (Table-1).

Seasonal abundance: During extensive survey in different localities of target area, it was observed during April to December. Its peak population was observed in November to December (Table-1).

Parasitoids : $B$. indicus and $L$. oregmae were recorded in the target area on this aphid with moderate to high rate of parasitisation.

\section{Predators :}

a. Coccinellids : C. sexmaculata, $C$. septempunctata, and $S$. pyrocheilus were recorded on this aphid with moderate to high rate of predation ( Table -1 ).

b. Syrphids : only $P$. serratus was recorded in the target area on A. nerii ( Table -1).

\section{APHIS PUNICAE}

It is small yellowish green aphid and commonly called Pomegranate aphid.

Distribution : It were recorded only in two localities of the target area.

Host Plants : It is not common aphid in the target area and were recorded on only two plants with low intensity of infestation (Colocacia sp. and Punica granatum).

Seasonal abundance : It was observed during October to November. 
Parasitoid : Not recorded.

Predator : Not recorded.

\section{APHIS SPIRAECOLA}

It is commonly known as spiraea aphid or apple aphid. It is small, bright greenish yellow or apple green in colour.

Distribution : It is a common aphid, generally found in almost all localities of the target area.

Host plants : It is a common aphid, generally found in all localities of the target area in abundant number and infest more than 18 food plants (Table-1). The most infested host plants were Brachycorne sp., Chrysanthemum indicum, Coriandrum sativum, F. vulgare and Trachyspermum roxburghianum. The moderate to low intensity of infestation was observed on rest of the plants. The most suffered family was Asteraceae.

Seasonal abundance: A. spiraecola was recorded during August to March and found abundantly during December to March.

Parasitoids : Aphilinus sp., A. gossypii, B. indicus and $L$. oregmae were recorded in the target area on this aphid with low to high rate of parasitisation (Table -1)

\section{Predators :}

a. Coccinellids : $C$. sexmaculata, $C$. septempunctata, $C$. transversalis and $S$. pyrocheilus were recorded on this aphid with moderate to high rate of predation (Table -1). b. Syrphids : only $P$. serratus was recorded in the target area on A. spiraecola (Table -1).

\section{ACKNOWLEDGEMENTS}

The authors are grateful to the head, University Department of Zoology, T. M. Bhagalpur University, Bhagalpur for providing facilities. We are also thankful to ZSI, Kolkata for identification of few species of predators.

\section{REFERENCES}

1. Agarwala, B.K. and A.K. Ghosh, 1988. Prey records of aphidophagous Coccinellidae in India. A review and bibliography. Trop. Pest Manage., 34: 1-14.

2. Agarwala, B.K., P. Laska and D.N. Raychaudhari, 1984. Prey records of aphidophagous syrphid flies from India (Diptera : Syrphidae). Acta Ent. Bohem., 81: 15-21

3. Ahmad, M.E. and N. Parween, 2009. New records of aphids and their parasitoids and hyperparasitiodes from northeast Bihar. $J$. Adv. Zool., 30(1): 7-9.

4. Ahmad, M.E. and R. Singh, 1996a. Records of aphid parasitoids from the north Bihar and associations with their hosts and food plants. J. Adv. Zool., 17: 26-33.

5. Ahmad, M.E. and R. Singh, 1996b. Trophic relations of aphid hyperparasitoids in northeastern Uttar Pradesh. Entomon, 21(1): 37 42.

6. Ahmad, M. E. and R. Singh, 1997. Records of aphids and their food plants, parasitoids and hyperparasitoids from the north Bihar. $J$. $A d v$. Zool., 18 (1) : 54 - 61.

7. Ahmad, M. E., K.M. Kumar, N. Parween and S. Kumar, 2009. Tetratrophic association of Aphis gossypii Glover with its food plants and natural enemies in northeast Bihar, pp. 
144-152. In : Eco-friendly Insect pest management (eds. Ignacimaithu and David), Elite pub. House.

8. Ahmad, M.E., N. Parween and S. Kumar, 2012. Taxo-ecological study of aphids infesting vegetable plants with their parasitoids in northeast Bihar. J. Appl. Zool. Res., 23(2): 103-114.

9. Behura, B.K., 1997. Bio-ecology of Indian aphids : a review. J. Aphidol., 11: 147-154.

10. Bisht, R.S., R.K. Sharma and Poonam Dev, 2006. Vertical distribution and activity of aphidophagous syrphidae (Diptera : Syrpdidae) in Garhwal Himalayas. $J$. Aphidol., 20(2): 25-29.

11. Blackman, R.L. and V.F. Eastop, 2000. Aphids on the world's crops : An identification and information guide, 2nd ed., John Wiley \& Sons, New York, pp. 466.

12. Chatha, A.M.M. and S. Naz, 2020. Effect of the parasitoids prey on prey consumption, survival, growth and development of Coccinella septempunctata L. (Coleoptera: Coccinellidae) under laboratory condition. Egyptian J. of Biological pest control, 30: 80

13. Dixon, A.F.G., 1985. Aphid Ecology. Blackie \& Sons Ltd., Bioshopbriggs, Glasgow, pp. 157.

14. Dutta, M., P. Parui and M. Mukerjee, 1997. Fauna of West Bengal. Zool. Sur. India. St. Fau. Series 3, Part 7: 1-76.

15. Ghorpade, K.D., 1981. Insect prey of Syrphidae (Diptera) from India and neighbouring countries; a review and bibiliography. Trop. Pest- Manag., 27: 62-82.

16. Ghorpade, K.D., 1994. Dignostic Keys to new and known genera and species of India subcontinent Syrphini (Diptera : Syrphidae). Colemania, 3: 1-15.

17. Ghosh, A.K. and B.K. Agarwala, 1982. A catalogue of Aphidiid (Hymenoptera: Aphidiidae) of Aphids (Homoptera) of India. J. Bombay Nat. Hist. Soc., 78: 124-134.

18. Gonazalez, D., F. Glistrap, P. Stray and L.
McKinnon, 1989. Foreign exploration for Russian wheat aphid and natural enemies : a summary of co-operative efforts by state University Agriculture Experiment Stations USDA/APHIS, USDA/ARS,CIBC, ICARDA and ITGC. Proc. Russian wheat Aphid Conference, Albuquerque, New Maxico. 113128.

19. Joshi, S., R.C. Ballal and N.S. Rao, 2000. The age-specific life-table of Paragus serratus (Fabr.) and Paragus yerburiensis STUCK (Diptera : Syrphidae), predators of Aphis craccivora Koch (Homoptera : Aphididae). J. Aphidol., 14: 67-72.

20. Joshi, S., T. Venkatesan and N.S. Rao, 1997. Host range and predatory fauna of Aphis craccivora Koch (Homoptera : Aphididae) in Bangalore, Karnataka. J. Bio. Contr., 11 : 59-63.

21. Kapur, A.P. and A.R. Bhaumik, 1961. A role on Ladybird beetle (Coleoptera : Coccinellidae) from Rajasthan with first record of Bulaea Lichatschovi (Hummel) from India. Rec. Indian Mus., 59: 445-448.

22. Kumar, A. and V.C. Kapoor, 1992. Collection and identification of hoverflies of East Punjab (India). J. Insect Sci., 5: 68-76.

23. Lingappa, S., R.K. Patil, P.S. Tippannavar, R.A. Balikai and Prasad Kumar, 2004. Development strategies for the management of woolly aphid, C. lanigera Z. (Homoptera : Aphidiadea) on sugarcane. Project report submitted to the Department of Agriculture, Government of Karnataka, April, 2004, pp. 46-53.

24. Omkar and R.B. Bind, 1993. Records of aphids natural enemies complex of Uttar- Pradesh. II. The Coccinellids. J. Adv. Zool., 14(2) : 96-99.

25. Omkar and R.B. Bind, 1995. Records of aphids natural enemies complex of Uttar- Pradesh. IV. The Coccinellids. J. Adv. Zool., 16: 6771.

26. Omkar and R.B. Bind, 1996. Records of aphids natural enemies complex of Uttar- Pradesh. 
V. The Coccinellids. J. Adv. Zool., 17: 4448.

27. Omkar and A. Pervez, 1999. New record of Coccinellids from Uttar Pradesh. I. J. $A d v$. Zool., 20: 106-112.

28. Omkar and A. Pervez, 2000. New record of Coccinellids from Uttar Pradesh. II. J. Adv. Zool., 21

29. Putra, N.S. and H. Yasuda, 2006. Effects of prey species and its density on larval performance of two species of Hoverfly larvae, Episyrphus balteatus de Geer and Eupeodes corollae Fabricius (Diptera : Syrphidae) Appl. Ent. Zool., 41: 389-397.

30. Radhakrishnan, B. and N. Muraleedharan, 1993. Bio-ecology of six species of syrphid predators of tea aphid, Toxoptera aurantii (Boyer de Fonscolombe) in Southern India. J. Entomon, 18: 175-180.

31. Raina, N., I.J. Samuel, Das and R. Singh, 2005. Feeding potential and its effect on development of an aphid predator, Episyrphus balteatus (De Geer) (Diptera : Syrphidae) vis-A-vis variable prey densities. J. Aphidol., 19: 93-100.

32. Rakhshan and Ahmad, 2019. Role of biological control agents in Integrated Pest Management approaches. Applied Entomology and Zoology volume 5 pp.69-88. Pub; Akinik pub. ISBN 978-93-5335-611-8

33. Raychaudhuri, D.N., 1980. Aphids of North-east India and Bhutan, pp. 459. Zool. Soc. Pub., Calcutta.

34. Sathe, T.V. and S. Visherad, 2004. Syrphids:As an important predator of aphids in India, In: Indian Insect Predator in Biological control (eds. K. Sahayaraj) pp. 261-273

35. Shantibala, K. and T.K. Singh, 1991. Studies on the vertical distribution of aphidophagous Coccinellids (Coleoptera : Coccinellidae) of
Manipur and Nagaland. J. Aphidol., 5: 3943.

36. Singh, R. and S.N. Rao, 1995. Biological control of Aphis gossypii Glover (Homoptera: Aphididae) on cucurbites by Trioxys indicus Subha Rao and Sharma (Hymenoptera : Aphididae) Bio. Agri. Hort., 12: 227-236.

37. Singh, R., B.S. Upadhaya, D. Singh and H.S. Chaudhary, 1999. Aphids (Homoptera: Aphididae) and their parasitoids in Northeast Uttar Pradesh. J. Aphidol., 13: 49-62.

38. Singh, K.C. and T.K. Singh, 1991. Aphidophagous Coccinellids of northeastern India Mizoram II. J. Adv. Zool., 12: 131134.

39. Stary, P. and A.K. Ghosh, 1979. Re-description of Trioxys nepalensis (Takada) and new host record of some aphids parasitoids (Hymenoptera: Aphidiidae) from Meghalaya, India. Orint. Insects, 13: 41-45.

40. Stary, P. and A.K. Ghosh, 1983. Aphis parasitoids of India and Adjacent Counteries (Hymenoptera : Aphidiidae). Tech. Monograph, 7. Zoological Survey of India, Calcutta, pp. 96 .

41. Subha Rao, B.R. and A.K. Sharma, 1962. Studies on the biology of Trioxys indicus Subha Rao and Sharma, a parasite of Aphis gossypii Glover. Proc. Nat. Inst. Sci. India 28: 164182.

42. Verma, A.K. and H.D. Makhmoor, 1987. Relative abundance of syrphid predators of Brevicoryne brassicae (L.) in cauliflower seed crop ecosystem. J. Biol. Contr., 1: 98103.

43. Volk, W., D.H. Stechman and P. Stary, 1990. Suitability of five species of Aphidiidae (Hymenoptera ) for the biological control of the banana aphid Pentalonia nigronervosa Coq. (Homoptera: Aphididae) in South Pacific. Trop. Pest. Manag., 36: 249- 257. 